\title{
Glossário LV: Um Plugin de Avaliação por Pares ao Glossário do Moodle
}

\author{
Manoel Fiuza L. Junior ${ }^{1}$, Gilvandenys L. Sales ${ }^{1}$, \\ Monck Charles N. Albuquerque ${ }^{2}$, Eliana A. Moreira Leite ${ }^{3}$ \\ ${ }^{1}$ Instituto Federal de Educação, Ciência e Tecnologia do Ceará - IFCE \\ Fortaleza - CE - Brasil \\ ${ }^{2}$ Instituto Federal de Educação, Ciência e Tecnologia da Bahia - IFBA \\ Seabra - BA - Brasil \\ ${ }^{3}$ Prefeitura Municipal de Fortaleza Ceará/Universidade do Minho Braga/Portugal \\ \{mfiuzajunior, denyssales, monckcna, elimoreiraead\}@gmail.com,
}

\begin{abstract}
Resumo. Este artigo apresenta o plugin Glossário LV, bem como sua integração ao modelo de avaliação Learning Vector (LV). Aplicando conceitos de avaliação por pares como metodologia ativa, o plugin permite que o aluno possa avaliar, dividindo com o professor essa tarefa. O Glossário LV foi desenvolvido de forma a facilitar o processo de avaliação por meios de ícones e sua integração com o Moodle é bem simples. Como resultado foi obtido um plugin de avaliação que deixa de ser centrada apenas no professor e passa a contar com a avaliação pelos alunos.
\end{abstract}

\begin{abstract}
This article introduces the LV Glossary plugin as well as its integration with the Learning Vector (LV) assessment model. Applying peer review concepts as an active methodology, the plugin allows the student to evaluate by sharing the task with the teacher. The LV Glossary was developed to facilitate the icon evaluation process and its integration with Moodle is very simple. As a result, an evaluation plugin was obtained that is no longer teacher-centered but now relies on student evaluation.
\end{abstract}

\section{Introdução}

As Tecnologias Digitais de Informação e Comunicação (TDIC) estão transformando nossa sociedade e a educação. Com o desenvolvimento dos Ambientes Virtuais de Aprendizagem (AVA), é possível fazer um curso cem por cento a distância. Há vários AVAs disponíveis, dentre eles podemos destaca: TelEduc, Amadeus, Dokeos, Sílabe e Moodle.

Dos AVAs citados, o Moodle é o mais utilizado no mundo, presente em mais de 179 países, distribuído de forma livre e gratuita. O Moodle disponibiliza uma grande quantidade de ferramentas avaliativas com suporte ao ensino presencial e a distância. Outra vantagem do Moodle é a facilidade de instalação e criação de plugins.

Com a preocupação de apresentar uma metodologia formativa de avaliação, foi criado um plugim para o Moodle chamado de Learning Vectors $(L V)$. O LV apresenta uma metodologia formativa de avaliação. São representações gráficas vetoriais concebidas para auxiliar o processo de avaliação em ambiente virtual de aprendizagem, considerando aspectos qualitativos e quantitativos no acompanhamento do desempenho do 
VIII Congresso Brasileiro de Informática na Educação (CBIE 2019)

Anais do XXV Workshop de Informática na Escola (WIE 2019)

aluno/usuário. Podem possibilitar feedbacks constantes das ações do aprendiz no AVA, além de reduzir a sobrecarga gerada para o Professor-Tutor em cursos a distância [Sales; Sales et al. 2010; 2012a].

O LV foi utilizado para acompanhar o desempenho de alunos que utilizam o AVA Moodle. As interações do aprendiz em fóruns, chats, wikis e tarefas são avaliadas não linearmente, através do uso de uma escala Likert de 5 pontos chamada de LV ícones. Os ícones passaram por uma atualização e foram transformados em Graphics Interchange Format (GIF), Gifs animados, na mediação da avaliação [Sales et al. 2019] conforme Figura 1.

\begin{tabular}{|c|c|c|c|c|c|c|}
\hline $\begin{array}{c}\text { Mençōes } \\
\text { Qualitativas }\end{array}$ & $\begin{array}{c}\text { Muito } \\
\text { bom }\end{array}$ & Bom & Regular & Fraco & $\begin{array}{c}\text { Não } \\
\text { Satisfatório }\end{array}$ & Neutro \\
\hline $\begin{array}{c}\text { Coeficiente do } \\
\text { Passo (CP) }\end{array}$ & 4 & 3 & 2 & 1 & 0 & - \\
\hline $\begin{array}{c}\text { LV } \\
\text { icones }\end{array}$ & -60 & -60 & 0 & 0 \\
\hline
\end{tabular}

Figura 1. Escala de Menções qualitativas e correspondência com o CP.

Outros autores como: [Prestes 2011], [Sales et al. 2012b], [Gonçalves 2016], [Marinho et al. 2016], [Albuquerque et al. 2017], têm desenvolvido pesquisas sobre o Learning Vectors (LV) e contribuído para os mecanismos de avaliações das seguintes atividades do Moodle: fóruns, chats, wikis e tarefas.

Uma das atividades do Moodle que ainda não foi estendida para avaliações com Learning Vectors foi o glossário. O glossário é um conjunto de termos e suas respectivas definições dentro de um determinado domínio do conhecimento.

Avaliar a inserção de registro no glossário e os seus comentários deixados pelos alunos é um requisito fundamental para promover orientação em meio à colaboração. Aproveitando que o Glossário possibilita essa colaboração Marinho et al. (2016) definiu uma modelagem do glossário do Moodle ao modelo de avaliação LV, chamando-o de "Glossário LV". As avaliações seguem conceitos dos LV aplicados às entradas criadas por alunos. No entanto, com o objetivo de aplicar a avaliação por pares como metodologia ativa, os comentários podem também receber notas que não devem vir do professor, mas sim de outros alunos.

A avaliação por pares não é um conceito recente. A comunicação científica tem tido contribuição dessa prática desde o século XVII, porém, só a partir da Segunda Guerra Mundial ela vem sendo formalizada [Mulligan et al. 2013].

Sugerida como metodologia ativa, esse tipo de avaliação visa aumentar o papel do estudante como agente principal no processo de sua formação, promovendo aprendizagem colaborativa. A condução do estudante para o centro da atuação na avaliação deve ser planejada pelo professor, que deve levantar e compartilhar os critérios de avaliação. Aperfeiçoar a autonomia do aluno tem como resultado um aumento de sua capacidade em compreender aspectos cognitivos, afetivos e culturais, desenvolvendo-o como um todo. 
VIII Congresso Brasileiro de Informática na Educação (CBIE 2019)

Anais do XXV Workshop de Informática na Escola (WIE 2019)

\section{Metodologia e Modelo do Glossário LV}

O Moodle apresenta diversas ferramentas disponíveis aos professores/tutores para trabalharem o ensino a distância, destaca-se neste trabalho de pesquisa o Glossário.

O glossário LV foi conceituado e modelado por Marinho et al. (2016). Este trabalho mostra como a atividade foi implementada e inserida como mais uma ferramenta a fazer parte do Plugin LV de avaliação [Sales et al. 2012b].

Há dois tipos de inserções que o aluno-aprendiz pode criar no glossário: entradas ou definições e comentários. O Professor-Tutor também pode criar entradas ou definições. Entradas e comentários podem ser avaliados. No entanto, apenas poderá avaliar, com uso da escala LV ícones, as entradas ou definições dos alunos-aprendizes.

As avaliações seguem as regras abaixo:

- O Professor-Tutor pode avaliar as definições, e apenas definições, criadas pelos alunos. Ele não pode avaliar comentários de qualquer natureza ou definições criadas por ele.

- Alunos podem avaliar comentários, e apenas comentários, que outros alunos deixam em entradas ou definições, exceto seus próprios comentários.

Apresenta-se na Figura 2 um exemplo de uma entrada criada por um aluno, vista do ponto de vista do Professor-Tutor.

\section{Galoneira}

Máquina utilizada para fazer acabamento em malha (barras simples, galão e trançador). Utilizada também na fabricação de lingerie para rebater elásticos, colocar viés, vivo e etc. São utilizadas duas linhas e um fio, e de uma a três agulhas, dependendo do acabamento que se quer ter.

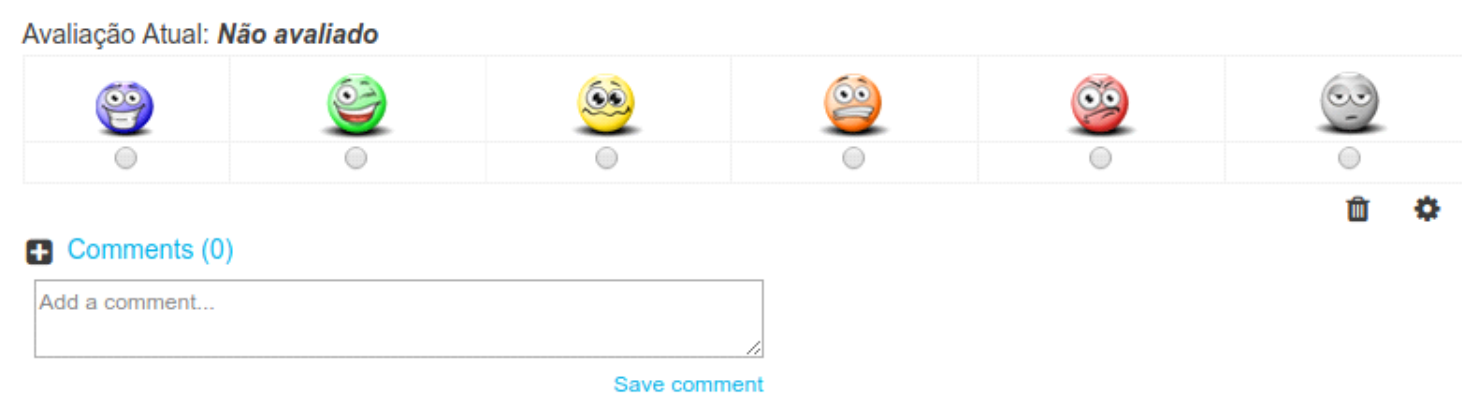

Figura 2. Interface do Glossário LV

Fonte:[Lima Junior et al. 2019]

A escala LV, que aparece logo abaixo da definição criada por um aluno, apenas será visível para o Professor-Tutor da disciplina para que ele possa realizar a avaliação. Ele também pode deixar algum comentário, caso deseje. O texto 'Não avaliado' aparece no lugar de uma nota não dada. Tão logo ele escolha um item da escala, a nota escolhida substituirá o texto. A nota dada será visível apenas paro o aluno avaliado e o professor avaliador. 
VIII Congresso Brasileiro de Informática na Educação (CBIE 2019)

Anais do XXV Workshop de Informática na Escola (WIE 2019)

Como afirmado, os comentários dos alunos para entradas criadas pelo professor ou outros alunos, também podem ser avaliados. Essa avaliação se dá apenas entre alunos, ou seja, o professor não é envolvido nas atribuições de notas dos comentários. A escala somente aparece abaixo de cada comentário criado por outros alunos, como mostrado na Figura 3:

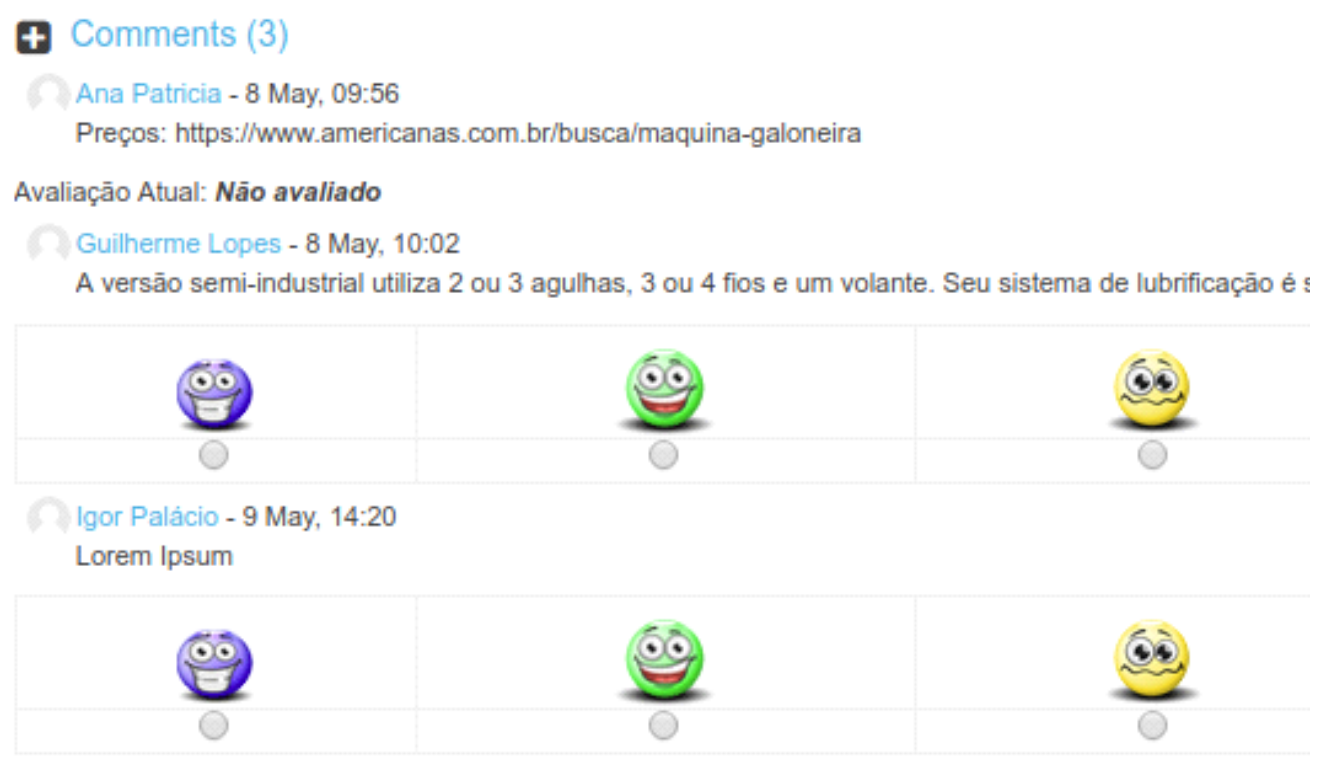

Figura 3. Interface de comentários em Glossário LV

Fonte:[Lima Junior et al. 2019]

Observa-se na Figura 3 que há três comentários. A aluna, autora da entrada, poderá avaliar os comentários deixados por outros alunos, apenas. Outros alunos poderão ver os comentários, e também avaliá-los. Somente o aluno que recebeu nota por seu comentário é capaz de visualizar a avaliação.

A primeira definição do aluno é classificada como termo principal (D) e avaliável apenas pelo Professor-Tutor. Seus comentários, classificados como complementos (C), somente podem ser avaliados por outros alunos, depois da primeira interação.

O Glossário LV foi integrado ao sistema de gerenciamento de notas do LV (SIG@LV). Atualmente, os módulos que foram estendidos e adaptados para receberem avaliações LV são: fórum, chat, wiki, tarefa e Glossário; cada módulo possui uma versão com LV que possibilita uma avaliação quantitativa e qualitativa do desempenho de alunos através de suas interações com atividades criadas no AVA.

\section{Considerações Finais}

O presente trabalho teve como objetivo apresentar as funcionalidades do plugin Glossário LV e sua utilização no Moodle. Foi apresentado seu funcionamento e demonstrado o processo de avaliação por pares. Esse tipo de avaliação visa aumentar o papel do estudante como agente principal no processo de sua formação, promovendo aprendizagem colaborativa suscitando o protagonismo dos estudantes no processo de ensino e aprendizagem.

O glossário LV proporciona ao discente a oportunidade de avaliar junto ao professor e faz com que o aluno olhe para seu próprio trabalho com outros olhos. Fazer com 
VIII Congresso Brasileiro de Informática na Educação (CBIE 2019)

Anais do XXV Workshop de Informática na Escola (WIE 2019)

que os alunos participem do processo de avaliação promovendo aprendizagem coletiva, uma vez que todos se veem como um grupo, ao passo que buscam os mesmos objetivos.

Por fim, como trabalho futuro, pretende-se realizar uma adaptação do glossário LV a gamificação na avaliação, para não só encorajá-los, mas também, trazer retorno lúdico, o que poderia fazer com que haja maior ímpeto em participar do processo avaliativo.

\section{Referências}

Albuquerque, M., Sales, G. L., Rebouças Filho, P., and Medeiros, C. (2017). Avaliação da presencialidade em um fórum lv utilizando lógica fuzzy. In Brazilian Symposium on Computers in Education (Simpósio Brasileiro de Informática na Educação-SBIE), volume 28, page 1357.

Gonçalves, A. J. (2016). Forum p\&r lv: avaliação formativa não-linear da aprendizagem aplicada ao lms moodle. Master's thesis, UNIVERSIDADE ESTADUAL DO CEARÁ - UECE.

Lima Junior, M. F., Sales, G. L., and Albuquerque, Monck Charles N. ans Leite, E. A. M. (2019). Plugin learning vectors aplicado à ferramenta glossário do moodle. In Anais dos Workshops do VIII Congresso Brasileiro de Informática na Educação (WCBIE).

Marinho, M. J. F. L., Cunha, J. L. L., Gonçalves, A. J., and Sales, G. L. (2016). Glossário hipertextual colaborativo lv. RENOTE, 14(1).

Mulligan, A., Hall, L., and Raphael, E. (2013). Peer review in a changing world: An international study measuring the attitudes of researchers. Journal of the American Society for Information Science and Technology, 64(1):132-161.

Prestes, P. A. N. (2011). Wiki-learning vectors ferramenta de avaliação da aprendizagem.

Sales, G., Albuquerque, M. C., Paz, R. P., and Leite, E. A. (2019). Modelo learning vectors na avaliação formativa no ava moodle: de emoticons a gifs animados. Revista Ibérica de Sistemas e Tecnologias de Informação, (E17):64-76.

Sales, G. L. (2010). Learning vectors (lv): um modelo de avaliação da aprendizagem em ead online aplicando métricas não-lineares. Tese de doutorado, Universidade Federal do Ceará-UFC.

Sales, G. L., Barroso, G. C., and Soares, J. M. (2012a). Learning vectors (lv): Um modelo de avaliação processual com mensuração não-linear da aprendizagem em ead online. Revista Brasileira de Informática na Educação, 20(1):60.

Sales, G. L., Leite, E. M., and Joye, C. R. (2012b). O sistema acadêmico lv: Avaliação em ead online implementada no ambiente virtual moodle. In Anais dos Workshops do Congresso Brasileiro de Informática na Educação, volume 1. 